\title{
Strong convergence and stability of implicit numerical methods for stochastic differential equations with non-globally Lipschitz continuous coefficients.
}

\author{
Xuerong Mao* $\quad$ Lukasz Szpruch ${ }^{\dagger}$
}

\begin{abstract}
We are interested in the strong convergence and almost sure stability of Euler-Maruyama (EM) type approximations to the solutions of stochastic differential equations (SDEs) with non-linear and nonLipschitzian coefficients. Motivation comes from finance and biology where many widely applied models do not satisfy the standard assumptions required for the strong convergence. In addition we examine the globally almost surely asymptotic stability in this non-linear setting for EM type schemes. In particular, we present a stochastic counterpart of the discrete LaSalle principle from which we deduce stability properties for numerical methods.
\end{abstract}

Key words: Dissipative model, super-linear growth, stochastic differential equation, strong convergence, backward Euler-Maruyama scheme, implicit method, LaSalle principle, non-linear stability, almost sure stability.

AMS Subject Clasification: 65C30, 65L20, 60H10

\section{Introduction}

Throughout this paper, let $\left(\Omega, \mathcal{F},\left\{\mathcal{F}_{t}\right\}_{t \geq 0}, \mathbb{P}\right)$ be a complete probability space with a filtration $\left\{\mathcal{F}_{t}\right\}_{t>0}$ satisfying the usual conditions (that is to say, it is right continuous and increasing while $\mathcal{F}_{0}$ contains all $\mathbb{P}$ null sets). Let $w(t)=\left(w_{1}(t), \ldots, w_{d}(t)\right)^{T}$ be a $d$-dimensional Brownian motion defined on the probability space, where $T$ denotes the transpose of a vector or a matrix. In this paper we study the numerical approximation of the stochastic differential equations (SDEs)

$$
d x(t)=f(x(t)) d t+g(x(t)) d w(t) .
$$

Here $x(t) \in \mathbb{R}^{n}$ for each $t \geq 0$ and $f: \mathbb{R}^{n} \rightarrow \mathbb{R}^{n}$ and $g: \mathbb{R}^{n} \rightarrow \mathbb{R}^{n \times d}$. For simplicity we assume that $x_{0} \in \mathbb{R}^{n}$ is a deterministic vector. Although the method of Lyapunov functions allows us to show that there are solutions to a very wide family of SDEs (see e.g. [24, 34]), in general, both the explicit solutions and the probability distributions of the solutions are not known. We therefore consider computable

\footnotetext{
${ }^{*}$ Department of Mathematics and Statistics, University of Strathclyde, Glasgow, G1 1XH, Scotland, UK (x.mao@strath.ac.uk).

${ }^{\dagger}$ Mathematical Institute, University of Oxford, Oxford, OX1 3LB, UK (lukas.szpruch@maths.ox.ac.uk).
} 
discrete approximations that, for example, could be used in Monte Carlo simulations. Convergence and stability of these methods are well understood for SDEs with Lipschitz continuous coefficients: see [26] for example. Our primary objective is to study classical strong convergence and stability questions for numerical approximations in the case where $f$ and $g$ are not globally Lipschitz continuous. A good motivation for our work is an instructive conditional result of Higham et al. [18. Under the local Lipschitz condition, they proved that uniform boundedness of moments of both the solution to (1.1) and its approximation are sufficient for strong convergence. That immediately raises the question of what type of conditions can guarantee such a uniform boundedness of moments. It is well known that the classical linear growth condition is sufficient to bound the moments for both the true solutions and their EM approximation 26, 34. It is also known that when we try to bound the moment of the true solutions, a useful way to relax the linear growth condition is to apply the Lyapunov-function technique, with $V(x)=\|x\|^{2}$. This leads us to the monotone condition [34. More precisely, if there exist constants $\alpha, \beta>0$ such that the coefficients of equation (1.1) satisfy

$$
\langle x, f(x)\rangle+\frac{1}{2}\|g(x)\|^{2} \leq \alpha+\beta\|x\|^{2} \quad \text { for all } x \in \mathbb{R}^{n}
$$

then

$$
\sup _{0 \leq t \leq T} \mathbb{E}\|x(t)\|^{2}<\infty \quad \forall T>0
$$

Here, and throughout, $\|x\|$ denotes both the Euclidean vector norm and the Frobenius matrix norm and $\langle x, y\rangle$ denotes the scalar product of vectors $x, y \in \mathbb{R}^{n}$. However, to the best of our knowledge there is no result on the moment bound for the numerical solutions of SDEs under the monotone condition (1.2). Additionally, Hutzenthaler et al. 23. proved that in the case of super-linearly growing coefficients, the EM approximation may not converge in the strong $L^{p}$-sense nor in the weak sense to the exact solution. For example, let us consider a non-linear SDE

$$
d x(t)=\left(\mu-\alpha x(t)^{3}\right) d t+\beta x(t)^{2} d w(t)
$$

where $\mu, \alpha, \beta \geq 0$ and $\alpha>\frac{1}{2} \beta^{2}$. In order to approximate SDE (1.4) numerically, for any $\Delta t$, we define the partition $\mathcal{P}_{\Delta t}:=\left\{t_{k}=k \Delta t: k=0,1,2, \ldots, N\right\}$ of the time interval $[0, T]$, where $N \Delta t=T$ and $T>0$. Then we define the EM approximation $Y_{t_{k}} \approx x\left(t_{k}\right)$ of (1.4) by

$$
Y_{t_{k+1}}=Y_{t_{k}}+\left(\mu-\alpha Y_{t_{k}}^{3}\right) \Delta t+\beta Y_{t_{k}}^{2} \Delta w_{t_{k}}
$$

where $\Delta w_{t_{k}}=w\left(t_{k+1}\right)-w\left(t_{k}\right)$. It was shown in [23] that

$$
\lim _{\Delta t \rightarrow 0} \mathbb{E}\left\|Y_{t_{N}}\right\|^{2}=\infty
$$

On the other hand, the coefficients of (1.4) satisfy the monotone condition (1.2) so (1.3) holds. Hence, Hutzenthaler et al. 23] concluded that

$$
\lim _{\Delta t \rightarrow 0} \mathbb{E}\left\|x(T)-Y_{t_{N}}\right\|^{2}=\infty
$$

It is now clear that to prove the strong convergence theorem under condition (1.2) it is necessary to modify the EM scheme. Motivated by the existing works [18] and 21] we consider implicit schemes. These authors have demonstrated that a backward Euler-Maruyama (BEM) method strongly converges to the solution of the SDE with one-sided Lipschitz drift and linearly growing diffusion coefficients. So far, to the best of our knowledge, most of the existing results on the strong convergence for numerical schemes only cover SDEs where the diffusion coefficients have at most linear growth [5, 39, 17, 22, 26. However, the problem remains essentially unsolved for the important class of SDEs with super-linearly growing diffusion coefficients. We are interested in relaxing the conditions for the diffusion coefficients to justify Monte Carlo simulations for highly non-linear systems that arise in financial mathematics, [1, 8, 2, 9, 14, 29], for example

$$
d x(t)=\left(\mu-\alpha x^{r}(t)\right) d t+\beta x^{\rho}(t) d w(t), \quad r, \rho>1,
$$


where $\mu, \alpha, \beta>0$, or in stochastic population dynamics [35, 3, 36, 41, 11], for example

$$
d x(t)=\operatorname{diag}\left(x_{1}(t), x_{2}(t), \ldots, x_{n}(t)\right)\left[\left(b+A x^{2}(t)\right) d t+x(t) d w(t)\right]
$$

where $b=\left(b_{1}, \ldots, b_{n}\right)^{T}, x^{2}(t)=\left(x_{1}^{2}(t), \ldots, x^{2}(t)_{n}\right)^{T}$ and matrix $A=\left[a_{i j}\right]_{1 \leq i, j \leq n}$ is such that $\lambda_{\max }(A+$ $\left.A^{T}\right)<0$, where $\lambda_{\max }(A)=\sup _{x \in \mathbb{R}^{n},\|x\|=1} x^{T} A x$. The only results we know, where the strong convergence of the numerical approximations was considered for super-linear diffusion, is Szpruch et al. [43. and Mao and Szpruch 37. In 43 authors have considered the BEM approximation for the following scalar SDE which arises in finance [2],

$$
d x(t)=\left(\alpha_{-1} x(t)^{-1}-\alpha_{0}+\alpha_{1} x(t)-\alpha_{2} x(t)^{r}\right) d t+\sigma x(t)^{\rho} d w(t) \quad r, \rho>1 .
$$

In 37, this analysis was extended to the multidimensional case under specific conditions for the drift and diffusion coefficients. In the present paper, we aim to prove strong convergence under general monotone condition (1.2) in a multidimensional setting. We believe that this condition is optimal for boundedness of moments of the implicit schemes. The reasons that we are interested in the strong convergence are: a) the efficient variance reduction techniques, for example, the multi-level Monte Carlo simulations [12], rely on the strong convergence properties; b) both weak convergence [26] and pathwise convergence [25] follow automatically.

Having established the strong convergence result we will proceed to the stability analysis of the underlying numerical scheme for the non-linear SDEs (1.1) under the monotone-type condition. The main problem concerns the propagation of an error during the simulation of an approximate path. If the numerical scheme is not stable, then the simulated path may diverge substantially from the exact solution in practical simulations. Similarly, the expectation of the functional estimated by a Monte Carlo simulation may be significantly different from that of the expected functional of the underlying SDEs due to numerical instability. Our aim here is to investigate almost surely asymptotic properties of the numerical schemes for SDEs (1.1) via a stochastic version of the LaSalle principle. LaSalle, 27], improved significantly the Lyapunov stability method for ordinary differential equations. Namely, he developed methods for locating limit sets of nonautonomous systems 13, 27. The first stochastic counterpart of his great achievement was established by Mao 33 under the local Lipschitz and linear growth conditions. Recently, this stochastic version was generalized by Shen et al. 42 to cover stochastic functional differential equations with locally Lipschitz continuous coefficients. Furthermore, it is well known that there exists a counterpart of the invariance principle for discrete dynamical systems [28]. However, there is no discrete stochastic counterpart of Mao's version of the LaSalle theorem. In this work we investigate a special case, with the Lyapunov function $V(x)=\|x\|^{2}$, of the LaSalle theorem. We shall show that the almost sure global stability can be easily deduced from our results. The primary objectives in our stability analysis are:

- Ability to cover highly nonlinear SDEs;

- Mild assumption on the time step - $A(\alpha)$-stability concept [15].

Results which investigate stability analysis for numerical methods can be found in Higham [16, 15. for the scalar linear case, Baker et al. 4] for the global Lipschitz and Higham et al. [19] for one-sided Lipschitz and the linear growth condition.

At this point, it is worth mentioning how our work compares with that of Higham et al. [18. Theorem 3.3 in their paper is a very important contribution to the numerical SDE theory. The authors proved strong convergence results for one-sided Lipschitz and the linear growth condition on drift and diffusion coefficients, respectively. What differentiates our work from [18 are: a) We significantly relax the linear growth constraint on the diffusion coefficient and we only ask for very general monotone type growth; b) Our analysis is based on a more widely applied BEM scheme in contrast to the split-step scheme introduced in their paper. An interesting alternative to the implicit schemes for numerical approximations of SDEs with non-globally Lipschitz drift coefficient recently appeared in 22. However the stability properties of this method are not analysed.

In what follows, for notational simplicity, we use the convention that $C$ represent a generic positive constant independent of $\Delta t$, the value of which may be different for different appearances. 
The rest of the paper is arranged as follows. In section 2, we introduce the monotone condition under which we prove the existence of a unique solution to equation (1.1), along with appropriate bounds that will be needed in further analysis. In Section 3 we propose the $\theta$-EM scheme, which is known as the BEM when $\theta=1$, to approximate the solution of equation (1.1). We show that the $2 n d$ moment of the $\theta$-EM, can be bounded under the monotone condition plus some mild assumptions on $f$ and $g$. In Section 4 we introduce a new numerical method, which we call the forward-backward Euler-Maruyama (FBEM). The FBEM scheme enables us to overcome some measurability difficulties and avoid using Malliavin calculus. We demonstrate that both the FBEM and the $\theta$-EM do not differ much in the $L^{p}$-sense. Then we prove a strong convergence theorem on a compact domain that is later extended to the whole domain. We also perform a numerical experiment that confirms our theoretical results. Section 5 contains the stability analysis, where we prove a special case of the stochastic LaSalle theorem for discrete time processes.

\section{Existence and Uniqueness of Solution}

We require the coefficients $f$ and $g$ in (1.1) to be locally Lipschitz continuous and to satisfy the monotone condition, that is

Assumption 2.1. Both coefficients $f$ and $g$ in (1.1) satisfy the following conditions:

Local Lipschitz condition. For each integer $m \geq 1$, there is a positive constant $C(m)$ such that

$$
\|f(x)-f(y)\|+\|g(x)-g(y)\| \leq C(m)\|x-y\|
$$

for those $x, y \in \mathbb{R}^{n}$ with $\|x\| \vee\|y\| \leq m$.

Monotone condition. There exist constants $\alpha$ and $\beta$ such that

$$
\langle x, f(x)\rangle+\frac{1}{2}\|g(x)\|^{2} \leq \alpha+\beta\|x\|^{2}
$$

for all $x \in \mathbb{R}^{n}$.

It is a classical result that under Assumption 2.1, there exists a unique solution to (1.1) for any given initial value $x(0)=x_{0} \in \mathbb{R}^{n},[10,34$. The reason why we present the following theorem with a proof here is that it reveals the upper bound for the probability that the process $x(t)$ stays on a compact domain for finite time $T>0$. The bound will be used to derive the main convergence theorem of this paper.

Theorem 2.2. Let Assumption 2.1 hold. Then for any given initial value $x(0)=x_{0} \in \mathbb{R}^{n}$, there exists a unique, global solution $\{x(t)\}_{t \geq 0}$ to equation (1.1). Moreover, the solution has the properties that for any $T>0$,

$$
\mathbb{E}\|x(T)\|^{2}<\left(\left\|x_{0}\right\|^{2}+2 \alpha T\right) \exp (2 \beta T)
$$

and

$$
\mathbb{P}\left(\tau_{m} \leq T\right) \leq \frac{\left(\left\|x_{0}\right\|^{2}+2 \alpha T\right) \exp (2 \beta T)}{m^{2}},
$$

where $m$ is any positive integer and

$$
\tau_{m}=\inf \{t \geq 0: \quad\|x(t)\|>m\}
$$

Proof. It is well known that under Assumption 2.1 for any given initial value $x_{0} \in \mathbb{R}^{n}$ there exists a unique solution $x(t)$ to the SDE (1.1), [?, 34]. Therefore we only need to prove that (2.2) and (2.3) hold. 
Applying the Itô formula to the function $V(x, t)=\|x\|^{2}$, we compute the diffusion operator

$$
L V(x, t)=2\left(\langle x, f(x)\rangle+\frac{1}{2}\|g(x)\|^{2}\right) .
$$

By Assumption 2.1

$$
L V(x, t) \leq 2 \alpha+2 \beta\|x\|^{2} .
$$

Therefore

$$
\mathbb{E}\left\|x\left(t \wedge \tau_{m}\right)\right\|^{2} \leq\left\|x_{0}\right\|^{2}+2 \alpha T+\int_{0}^{t} 2 \beta \mathbb{E}\left\|x\left(s \wedge \tau_{m}\right)\right\|^{2} d s .
$$

The Gronwall inequality gives

$$
\mathbb{E}\left\|x\left(T \wedge \tau_{m}\right)\right\|^{2} \leq\left(\left\|x_{0}\right\|^{2}+2 \alpha T\right) \exp (2 \beta T) .
$$

Hence

$$
\mathbb{P}\left(\tau_{m} \leq T\right) m^{2} \leq\left[\left\|x_{0}\right\|^{2}+2 \alpha T\right] \exp (2 \beta T) .
$$

Next, letting $m \rightarrow \infty$ in (2.6) and applying Fatou's lemma, we obtain

$$
\mathbb{E}\|x(T)\|^{2} \leq\left[\left\|x_{0}\right\|^{2}+2 \alpha T\right] \exp (2 \beta T),
$$

which gives the other assertion (2.2) and completes the proof.

\section{The $\theta$-Euler-Maruyama Scheme}

As indicated in the introduction, in order to approximate the solution of 1.1) we will use the $\theta$-EM scheme. Given any step size $\Delta t$, we define a partition $\mathcal{P}_{\Delta t}:=\left\{t_{k}=k \Delta t: k=0,1,2, \ldots\right\}$ of the half line $[0, \infty)$, and define

$$
X_{t_{k+1}}=X_{t_{k}}+\theta f\left(X_{t_{k+1}}\right) \Delta t+(1-\theta) f\left(X_{t_{k}}\right) \Delta t+g\left(X_{t_{k}}\right) \Delta w_{t_{k}},
$$

where $\Delta w_{t_{k}}=w_{t_{k+1}}-w_{t_{k}}$ and $X_{t_{0}}=x_{0}$. The additional parameter $\theta \in[0,1]$ allows us to control the implicitness of the numerical scheme, that may lead to various asymptotic behaviours of equation (3.1). For technical reasons we always require $\theta \geq 0.5$.

Since we are dealing with an implicit scheme we need to make sure that equation (3.1) has a unique solution $X_{t_{k+1}}$ given $X_{t_{k}}$. To prove this, in addition to Assumption 2.1, we ask that function $f$ satisfies the one-sided Lipschitz condition.

Assumption 3.1. One-sided Lipschitz condition. There exists a constant $L>0$ such that

$$
\langle x-y, f(x)-f(y)\rangle \leq L\|x-y\|^{2} \quad \forall x, y \in \mathbb{R}^{n} .
$$

It follows from the fixed point theorem that a unique solution $X_{t_{k+1}}$ to equation (3.1) exists given $X_{t_{k}}$, provided $\Delta t<\frac{1}{\theta L}$, (see [37] for more details). From now on we always assume that $\Delta t<\frac{1}{\theta L}$. In order to implement numerical scheme (3.1) we define a function $F: \mathbb{R}^{n} \rightarrow \mathbb{R}^{n}$ as

$$
F(x)=x-\theta f(x) \Delta t
$$

Due to Assumption 3.1, there exists an inverse function $F^{-1}$ and the solution to (3.1) can be represented 
in the following form

$$
X_{t_{k+1}}=F^{-1}\left(X_{t_{k}}+(1-\theta) f\left(X_{t_{k}}\right) \Delta t+g\left(X_{t_{k}}\right) \Delta w_{t_{k}}\right)
$$

Clearly, $X_{t_{k}}$ is $\mathcal{F}_{t_{k}}$-measurable. In many applications, the drift coefficient of the SDEs has a cubic or quadratic form, whence the inverse function can be found explicitly. For more complicated SDEs we can find the inverse function $F^{-1}$ using root-finding algorithms, such as Newton's method.

\subsection{Moment Properties of $\theta$-EM}

In this section we show that the second moment of the $\theta$-EM (3.1) is bounded (Theorem 3.6). To achieve the bound we employ the stopping time technique, in a similar way as in the proof of Theorem 2.2 . However, in discrete time approximations for a stochastic process, the problem of overshooting the level where we would like to stop the process appears, [7, 6, 32.

Due to the implicitness of scheme (3.1), an additional but mild restriction on the time step appears. That is, from now on, we require $\Delta t \leq \Delta t^{*}$, where $\Delta t^{*} \in\left(0,(\max \{L, 2 \beta\} \theta)^{-1}\right)$ with $\beta$ and $L$ defined in Assumptions 2.1 and 3.1 respectively.

The following lemma shows that in order to guarantee the boundedness of moments for $X_{t_{k}}$ defined by (3.1) it is enough to bound the moments of $F\left(X_{t_{k}}\right)$, where $F$ is defined by (3.2).

Lemma 3.2. Let Assumption 2.1 hold. Then for $F(x)=x-\theta f(x) \Delta t$ we have

$$
\|x\|^{2} \leq(1-2 \beta \theta \Delta t)^{-1}\left[\|F(x)\|^{2}+2 \theta \alpha \Delta t\right] \quad \forall x \in \mathbb{R}^{n} .
$$

Proof. Writing $\|F(x)\|^{2}=\langle F(x), F(x)\rangle$ and using Assumption 2.1 we arrive at

$$
\begin{aligned}
\|F(x)\|^{2} & =\|x\|^{2}-2 \theta\langle x, f(x)\rangle \Delta t+\theta^{2}\|f(x)\|^{2} \Delta t^{2} \\
& \geq(1-2 \beta \theta \Delta t)\|x\|^{2}-2 \theta \alpha \Delta t,
\end{aligned}
$$

and the assertion follows.

We define the stopping time $\lambda_{m}$ by

$$
\lambda_{m}=\inf \left\{k:\left\|X_{t_{k}}\right\|>m\right\}
$$

We observe that when $k \in\left[0, \lambda_{m}(\omega)\right],\left\|X_{t_{k-1}}(\omega)\right\| \leq m$, but we may have $\left\|X_{t_{k}}(\omega)\right\|>m$, so the following lemma is not trivial.

Lemma 3.3. Let Assumptions [2.1, 3.1] hold, and $\theta \geq 0,5$. Then for $p \geq 2$ and sufficiently large integer $m$, there exists a constant $C(p, m)$, such that

$$
\mathbb{E}\left[\left\|X_{t_{k}}\right\|^{p} \mathbf{1}_{\left[0, \lambda_{m}\right]}(k)\right]<C(p, m) \quad \text { for any } k \geq 0
$$

Proof. The proof is given in the Appendix.

For completeness of the exposition we recall the discrete Gronwall inequality, that we will use in the proof of Theorem 3.6 . 
Lemma 3.4 (The Discrete Gronwall Inequality). Let $M$ be a positive integer. Let $u_{k}$ and $v_{k}$ be nonnegative numbers for $k=0,1, \ldots, M$. If

$$
u_{k} \leq u_{0}+\sum_{j=0}^{k-1} v_{j} u_{j}, \quad \forall k=1,2, \ldots, M,
$$

then

$$
u_{k} \leq u_{0} \exp \left(\sum_{j=0}^{k-1} v_{j}\right), \quad \forall k=1,2, \ldots, M
$$

The proof can be found in Mao et al. [38. To prove the boundedness of the second moment for the $\theta$-EM (3.1), we need an additional but mild assumption on the coefficients $f$ and $g$.

Assumption 3.5. The coefficients of equation (1.1) satisfy the polynomial growth condition. That is, there exists a pair of constants $h \geq 1$ and $C(h)>0$ such that

$$
\|f(x)\| \vee\|g(x)\| \leq C(h)\left(1+\|x\|^{h}\right), \quad \forall x \in \mathbb{R}^{n} .
$$

Let us begin to establish the fundamental result of this paper that reveals the boundedness of the second moments for SDEs (1.1) under Assumptions 2.1 and 3.5 .

Theorem 3.6. Let Assumptions 2.1, 3.1, 3.5 hold, and $\theta \geq 0.5$. Then, for any $T>0$, there exists a constant $C(T)>0$, such that the $\theta$-EM scheme (3.1) has the following property

$$
\sup _{\Delta t \leq \Delta t^{*}} \sup _{0 \leq t_{k} \leq T} \mathbb{E}\left\|X_{t_{k}}\right\|^{2}<C(T) .
$$

Proof. By definition (3.2) of function $F$, we can represent the $\theta$-EM scheme (3.1) as

$$
F\left(X_{t_{k+1}}\right)=F\left(X_{t_{k}}\right)+f\left(X_{t_{k}}\right) \Delta t+g\left(X_{t_{k}}\right) \Delta w_{t_{k}} .
$$

Consequently writing $\left\langle F\left(X_{t_{k+1}}\right), F\left(X_{t_{k+1}}\right)\right\rangle=\left\|F\left(X_{t_{k+1}}\right)\right\|^{2}$ and utilizing Assumption 2.1] we obtain

$$
\begin{aligned}
\left\|F\left(X_{t_{k+1}}\right)\right\|^{2}= & \left\|F\left(X_{t_{k}}\right)\right\|^{2}+\left\|f\left(X_{t_{k}}\right)\right\|^{2} \Delta t^{2}+\left\|g\left(X_{t_{k}}\right)\right\|^{2} \Delta t \\
& +2\left\langle F\left(X_{t_{k}}\right), f\left(X_{t_{k}}\right)\right\rangle \Delta t+\Delta M_{t_{k+1}} \\
= & \left\|F\left(X_{t_{k}}\right)\right\|^{2} \\
& +\left(2\left\langle X_{t_{k}}, f\left(X_{t_{k}}\right)\right\rangle+\left\|g\left(X_{t_{k}}\right)\right\|^{2}\right) \Delta t \\
& +(1-2 \theta)\left\|f\left(X_{t_{k}}\right)\right\|^{2} \Delta t^{2}+\Delta M_{t_{k+1}}
\end{aligned}
$$

where

$$
\begin{aligned}
\Delta M_{t_{k+1}} & =\left\|g\left(X_{t_{k}}\right) \Delta w_{t_{k+1}}\right\|^{2}-\left\|g\left(X_{t_{k}}\right)\right\|^{2} \Delta t+2\left\langle F\left(X_{t_{k}}\right), g\left(X_{t_{k}}\right) \Delta w_{t_{k+1}}\right\rangle \\
& +2\left\langle f\left(X_{t_{k}}\right) \Delta t, g\left(X_{t_{k}}\right) \Delta w_{t_{k+1}}\right\rangle,
\end{aligned}
$$

is a local martingale. By Assumption 2.1 and the fact that $\theta \geq 0.5$,

$$
\left\|F\left(X_{t_{k+1}}\right)\right\|^{2} \leq\left\|F\left(X_{t_{k}}\right)\right\|^{2}+2 \alpha \Delta t+2 \beta\left\|X_{t_{k}}\right\|^{2} \Delta t+\Delta M_{t_{k+1}} .
$$


Let $N$ be any nonnegative integer such that $N \Delta t \leq T$. Summing up both sides of inequality (3.6) from $k=0$ to $N \wedge \lambda_{m}$, we get

$$
\begin{aligned}
\left\|F\left(X_{t_{N \wedge \lambda_{m}+1}}\right)\right\|^{2} & \leq\left\|F\left(X_{t_{0}}\right)\right\|^{2}+2 \alpha T+2 \beta \sum_{k=0}^{N \wedge \lambda_{m}}\left\|X_{t_{k}}\right\|^{2} \Delta t+\sum_{k=0}^{N \wedge \lambda_{m}} \Delta M_{t_{k+1}} \\
& \leq\left\|F\left(X_{t_{0}}\right)\right\|^{2}+2 \alpha T+2 \beta \sum_{k=0}^{N}\left\|X_{t_{k \wedge \lambda m}}\right\|^{2} \Delta t+\sum_{k=0}^{N} \Delta M_{t_{k+1}} \mathbf{1}_{\left[0, \lambda_{m}\right]}(k) .
\end{aligned}
$$

Applying Lemma 3.3. Assumption 3.5] and noting that $X_{t_{k}}$ and $\mathbf{1}_{\left[0, \lambda_{m}\right]}(k)$ are $\mathcal{F}_{t_{k}}$-measurable while $\Delta w_{t_{k}}$ is independent of $\mathcal{F}_{t_{k}}$, we can take expectations on both sides of (3.7) to get

$$
\mathbb{E}\left\|F\left(X_{t_{N \wedge \lambda_{m}+1}}\right)\right\|^{2} \leq\left\|F\left(X_{t_{0}}\right)\right\|^{2}+2 \alpha T+2 \beta \mathbb{E}\left[\sum_{k=0}^{N}\left\|X_{t_{k \wedge \lambda m}}\right\|^{2} \Delta t\right]
$$

By Lemma 3.2

$$
\begin{aligned}
\mathbb{E}\left\|F\left(X_{t_{N \wedge \lambda_{m}+1}}\right)\right\|^{2} \leq & \left\|F\left(X_{t_{0}}\right)\right\|^{2}+\left(2 \alpha+2 \beta(1-2 \beta \theta \Delta t)^{-1} 2 \theta \alpha \Delta t\right)(T+\Delta t) \\
& +2 \beta(1-2 \beta \theta \Delta t)^{-1} \mathbb{E}\left[\sum_{k=0}^{N}\left\|F\left(X\left(t_{k \wedge \lambda_{m}}\right)\right)\right\|^{2} \Delta t\right]
\end{aligned}
$$

By the discrete Gronwall inequality

$\mathbb{E}\left\|F\left(X_{t_{N \wedge \lambda m+1}}\right)\right\|^{2} \leq\left[\left\|F\left(X_{t_{0}}\right)\right\|^{2}+\left(2 \alpha+2 \beta(1-2 \beta \theta \Delta t)^{-1} 2 \theta \alpha \Delta t\right)(T+\Delta t)\right] \exp \left(2 \beta(1-2 \beta \theta \Delta t)^{-1}(T+\Delta t)\right)$,

where we use the fact that $N \Delta t \leq T$. Thus, letting $m \rightarrow \infty$ in (3.8) and applying Fatou's lemma, we get

$$
\mathbb{E}\left\|F\left(X_{t_{N+1}}\right)\right\|^{2} \leq\left[\left\|F\left(X_{t_{0}}\right)\right\|^{2}+\left(2 \alpha+2 \beta(1-2 \beta \theta \Delta t)^{-1} 2 \theta \alpha \Delta t\right)(T+\Delta t)\right] \exp \left(2 \beta(1-2 \beta \theta \Delta t)^{-1}(T+\Delta t)\right) .
$$

By Lemma 3.2, the proof is complete.

\section{Forward-Backward Euler-Maruyama Scheme}

We find in our analysis that it is convenient to work with a continuous extension of a numerical method. This continuous extension enables us to use the powerful continuous-time stochastic analysis in order to formulate theorems on numerical approximations. We find it particularly useful in the proof of forthcoming Theorem 4.2, Let us define

$$
\begin{gathered}
\eta(t):=t_{k}, \quad \text { for } \quad t \in\left[t_{k}, t_{k+1}\right), k \geq 0, \\
\eta_{+}(t):=t_{k+1}, \quad \text { for } \quad t \in\left[t_{k}, t_{k+1}\right), k \geq 0 .
\end{gathered}
$$

One possible continuous version of the $\theta$-EM is given by

$$
X(t)=X_{t_{0}}+\theta \int_{0}^{t} f\left(X_{\eta_{+}(s)}\right) d s+(1-\theta) \int_{0}^{t} f\left(X_{\eta(s)}\right) d s+\int_{0}^{t} g\left(X_{\eta(s)}\right) d w(s), \quad t \geq 0 .
$$


Unfortunately, this $X(t)$ is not $\mathcal{F}_{t}$-adapted whence it does not meet the fundamental requirement in the classical stochastic analysis. To avoid using Malliavin calculus, we introduce a new numerical scheme, which we call the Forward-Backward Euler-Maruyama (FBEM) scheme: Once we compute the discrete values $X_{t_{k}}$ from the $\theta$-EM, that is

$$
X_{t_{k}}=X_{t_{k-1}}+\theta f\left(X_{t_{k}}\right) \Delta t+(1-\theta) f\left(X_{t_{k-1}}\right) \Delta t+g\left(X_{t_{k-1}}\right) \Delta w_{t_{k-1}},
$$

we define the discrete FBEM by

$$
\hat{X}_{t_{k+1}}=\hat{X}_{t_{k}}+f\left(X_{t_{k}}\right) \Delta t+g\left(X_{t_{k}}\right) \Delta w_{t_{k}}
$$

where $\hat{X}_{t_{0}}=X_{t_{0}}=x_{0}$, and the continuous FBEM by

$$
\hat{X}(t)=\hat{X}_{t_{0}}+\int_{0}^{t} f\left(X_{\eta(s)}\right) d s+\int_{0}^{t} g\left(X_{\eta(s)}\right) d w(s), \quad t \geq 0 .
$$

Note that the continuous and discrete BFEM schemes coincide at the gridpoints; that is, $\hat{X}\left(t_{k}\right)=\hat{X}_{t_{k}}$.

\subsection{Strong Convergence on the Compact Domain}

It this section we prove the strong convergence theorem. We begin by showing that both schemes of the FBEM (4.2) and the $\theta$-EM (3.1) stay close to each other on a compact domain. Then we estimate the probability that both continuous FBEM (4.3) and $\theta$-EM (3.1) will not explode on a finite time interval.

Lemma 4.1. Let Assumptions 2.1, 3.1, 3.5 hold, and $\theta \geq 0.5$. Then for any integer $p \geq 2$ and $m \geq\left\|x_{0}\right\|$, there exists a constant $C(m, p)$ such that

$$
\mathbb{E}\left[\left\|\hat{X}_{t_{k}}-X_{t_{k}}\right\|^{p} \mathbf{1}_{\left[0, \lambda_{m}\right]}(k)\right] \leq C(m, p) \Delta t^{p}, \quad \forall k \in \mathbb{N}
$$

and for $F(x)=x-\theta f(x) \Delta t$ we have

$$
\left\|\hat{X}_{t_{k}}\right\|^{2} \geq \frac{1}{2}\left\|F\left(X_{t_{k}}\right)\right\|^{2}-\left\|\theta f\left(x_{0}\right) \Delta t\right\|^{2} \quad \forall k \in \mathbb{N} .
$$

Proof. Summing up both schemes of the FBEM (4.2) and the $\theta$-EM (3.1), respectively, we obtain

$$
\hat{X}_{t_{N}}-X_{t_{N}}=\theta\left(f\left(X_{t_{0}}\right)-f\left(X_{t_{N}}\right)\right) \Delta t
$$

By Hölder's inequality, Lemma 3.3 and Assumption 3.5 , we then see easily that there exists a constant $C(m, p)>0$, such that

$$
\mathbb{E}\left[\left\|\hat{X}_{t_{N}}-X_{t_{N}}\right\|^{p} \mathbf{1}_{\left[0, \lambda_{m}\right]}(N)\right]=\theta \mathbb{E}\left[\left\|f\left(X_{t_{0}}\right) \Delta t-f\left(X_{t_{N}}\right) \Delta t\right\|^{p} \mathbf{1}_{\left[0, \lambda_{m}\right]}(N)\right] \leq C(m, p) \Delta t^{p}
$$

as required. Next, using inequality $2|a||b| \leq \varepsilon|a|^{2}+\varepsilon^{-1}|b|^{2}$ with $\varepsilon=0.5$ we arrive at

$$
\begin{aligned}
\left\|\hat{X}_{t_{N}}\right\|^{2} & =\left\|X_{t_{N}}-\theta f\left(X_{t_{N}}\right) \Delta t+\theta f\left(X_{t_{0}}\right) \Delta t\right\|^{2} \geq\left(\left\|F\left(X_{t_{N}}\right)\right\|-\left\|\theta f\left(X_{t_{0}}\right) \Delta t\right\|\right)^{2} \\
& \geq \frac{1}{2}\left\|F\left(X_{t_{N}}\right)\right\|^{2}-\left\|\theta f\left(X_{t_{0}}\right) \Delta t\right\|^{2}
\end{aligned}
$$


The following Theorem provides us with a similar estimate for the distribution of the first passage time for the continuous FBEM (4.3) and $\theta$-EM (3.1) as we have obtained for the SDEs (1.1) in Theorem 2.2. We will use this estimate in the proof of forthcoming Theorem 4.4 .

Theorem 4.2. Let Assumptions 2.1, 3.1, 3.5 hold, and $\theta \geq 0.5$. Then, for any given $\epsilon>0$, there exists a positive integer $N_{0}$ such that for every $m \geq N_{0}$, we can find a positive number $\Delta t_{0}=\Delta t_{0}(m)$ so that whenever $\Delta t \leq \Delta t_{0}$,

$$
\mathbb{P}\left(\vartheta_{m}<T\right) \leq \epsilon, \quad \text { for } T>0,
$$

where $\vartheta_{m}=\inf \left\{t>0:\|\hat{X}(t)\| \geq m\right.$ or $\left.\left\|X_{\eta(t)}\right\|>m\right\}$.

Proof. The proof is given in the Appendix.

\subsection{Strong Convergence on the Whole Domain}

In this section we present the main theorem of this paper, the strong convergence of the $\theta$-EM (3.1) to the solution of (1.1). First, we will show that the continuous FBEM (4.3) converges to the true solution on the compact domain. This, together with Theorem 4.2 will enable us to extend convergence to the whole domain. Let us define the stopping time

$$
\theta_{m}=\tau_{m} \wedge \vartheta_{m},
$$

where $\tau_{m}$ and $\vartheta_{m}$ are defined in Theorems 2.2 and 4.2 , respectively.

Lemma 4.3. Let Assumptions 2.1, 3.1, 3.5 hold, and $\theta \geq 0.5$. For sufficiently large $m$, there exists a positive constant $C(T, m)$, such that

$$
\mathbb{E}\left[\sup _{0 \leq t \leq T}\left\|\hat{X}\left(t \wedge \theta_{m}\right)-x\left(t \wedge \theta_{m}\right)\right\|^{2}\right] \leq C(T, m) \Delta t .
$$

Proof. The proof is given in the Appendix.

We are now ready to prove the strong convergence of the $\theta$-EM (3.1) to the true solution of (1.1).

Theorem 4.4. Let Assumptions 2.1, 3.1, 3.5 hold, and $\theta \geq 0.5$. For any given $T=N \Delta t>0$ and $s \in[1,2), \theta$-EM scheme (3.1) has the property

$$
\lim _{\Delta t \rightarrow 0} \mathbb{E}\left\|X_{T}-x(T)\right\|^{s}=0 .
$$

Proof. Let

$$
e(T)=X_{T}-x(T) .
$$

Applying Young's inequality

$$
x^{s} y \leq \frac{\delta s}{2} x^{2}+\frac{2-s}{2 \delta^{\frac{s}{2-s}}} y^{\frac{2}{2-s}}, \quad \forall x, y, \delta>0,
$$


leads us to

$$
\begin{aligned}
\mathbb{E}\|e(T)\|^{s} & \left.=\mathbb{E}\left[\|e(T)\|^{s} \mathbf{1}_{\left\{\tau_{m}>T, \vartheta_{m}>T\right\}}\right]+\mathbb{E}\left[\|e(T)\|^{s} \mathbf{1}_{\left\{\tau_{m} \leq T\right.} \quad \text { or } \quad \vartheta_{m} \leq T\right\}\right] \\
& \leq 2^{s-1}\left[\mathbb{E}\left[\|\hat{X}(T)-x(T)\|^{s} \mathbf{1}_{\left\{\tau_{m}>T, \vartheta_{m}>T\right\}}\right]+\mathbb{E}\left[\left\|X_{T}-\hat{X}(T)\right\|^{s} \mathbf{1}_{\left\{\tau_{m}>T, \vartheta_{m}>T\right\}}\right]\right] \\
& +\frac{\delta s}{2} \mathbb{E}\left[\|e(T)\|^{2}\right]+\frac{2-s}{2 \delta^{\frac{s}{2-s}}} \mathbb{P}\left(\tau_{m} \leq T \quad \text { or } \quad \vartheta_{m} \leq T\right) .
\end{aligned}
$$

First, let us observe that by Lemma 4.1 we obtain

$$
\mathbb{E}\left[\left\|X_{T}-\hat{X}(T)\right\|^{s} \mathbf{1}_{\left\{\tau_{m}>T, \vartheta_{m}>T\right\}}\right] \leq C(m, s) \Delta t^{s}
$$

Given an $\epsilon>0$, by Hölder's inequality and Theorems 2.2 and 3.6 , we choose $\delta$ such that

$$
\frac{\delta s}{2} \mathbb{E}\left[\|e(T)\|^{2}\right] \leq \delta s \mathbb{E}\left[\|x(T)\|^{2}+\left\|X_{T}\right\|^{2}\right] \leq \frac{\epsilon}{3}
$$

Now by (2.3) there exists $N_{0}$ such that for $m \geq N_{0}$

$$
\frac{2-s}{2 \delta^{\frac{s}{2-s}}} \mathbb{P}\left(\tau_{m} \leq T\right) \leq \frac{\epsilon}{3}
$$

and finally by Lemmas 4.1, 4.3 and Theorem 4.2 we choose $\Delta t$ sufficiently small, such that

$2^{s-1}\left[\mathbb{E}\left[\|\hat{X}(T)-x(T)\|^{s} \mathbf{1}_{\left\{\tau_{m}>T, \vartheta_{m}>T\right\}}\right]+\mathbb{E}\left[\left\|X_{T}-\hat{X}(T)\right\|^{s} \mathbf{1}_{\left\{\tau_{m}>T, \vartheta_{m}>T\right\}}\right]\right]+\frac{2-s}{2 \delta^{\frac{s}{2-s}}} \mathbb{P}\left(\vartheta_{m} \leq T\right) \leq \frac{\epsilon}{3}$

which completes the proof.

Theorem 4.4 covers many highly non-linear SDEs, though it might be computationally expensive to find the inverse $F^{-1}$ of the function $F(x)=x-\theta f(x) \Delta t$. For example, lets consider equation (1.4) with $\mu(x)=a+\sin (x)^{2}, a>0$, that is

$$
d x(t)=\left(a+\sin (x)^{2}-\alpha x(t)^{3}\right) d t+\beta x(t)^{2} d w(t)
$$

where $\alpha, \beta>0$. This type of SDE could be used to model electricity prices where we need to account for a seasonality pattern, 31. In this situation, it is useful to split the drift coefficient in two parts, that is

$$
f(x)=f_{1}(x)+f_{2}(x)
$$

This allows us to introduce partial implicitness in the numerical scheme. In the case of (4.7) we would take $f_{1}(x)=-\alpha x(t)^{3}$ and $f_{2}(x)=a+\sin (x)^{2}$. Then a new partially implicit $\theta$-EM scheme has the following form

$$
Y_{t_{k+1}}=Y_{t_{k}}+\theta f_{1}\left(Y_{t_{k+1}}\right) \Delta t+(1-\theta) f_{1}\left(Y_{t_{k}}\right) \Delta t+f_{2}\left(Y_{t_{k}}\right) \Delta t+g\left(Y_{t_{k}}\right) \Delta w_{t_{k}}
$$

It is enough that $f_{1}$ satisfies Assumption 3.1 in order for scheme (4.9) to be well defined. Its solution can be represented as

$$
Y_{t_{k+1}}=H^{-1}\left(Y_{t_{k}}+(1-\theta) f_{1}\left(Y_{t_{k}}\right) \Delta t+f_{2}\left(Y_{t_{k}}\right) \Delta t+g\left(Y_{t_{k}}\right) \Delta w_{t_{k}}\right)
$$

where

$$
H(x)=x-\theta f_{1}(x) \Delta t
$$

All results from Sections 3 and 4 hold, once we replace condition (2.1) in Assumption 2.1 and Assumption 3.1 by (4.11) (4.12), respectively. 
Theorem 4.5. Let Assumption 3.5 hold and $\Delta t \in\left[0,(\max \{L, 2 \beta\} \theta)^{-1}\right)$. In addition we assume that for $x, y \in \mathbb{R}^{n}$, there exist constants $L, \alpha, \beta>0$ such that

$$
\left\langle x-y, f_{1}(x)-f_{1}(y)\right\rangle \leq L\|x-y\|^{2},
$$

and

$$
\langle x, f(x)\rangle+\frac{1}{2}\|g(x)\|^{2}+\left[(1-\theta)\left\langle f_{1}(x), f_{2}(x)\right\rangle+\frac{1}{2}\left\|f_{2}(x)\right\|^{2}+\frac{1}{2}(1-2 \theta)\left\|f_{1}(x)\right\|^{2}\right] \Delta t \leq \alpha+\beta\|x\|^{2} .
$$

Then for any given $T>0$ and $s \in[1,2), \theta$-EM scheme (4.9) has the following property

$$
\lim _{\Delta t \rightarrow 0} \mathbb{E}\left\|Y_{T}-x(T)\right\|^{s}=0
$$

Proof. In order to prove Theorem4.5 we need to show that results from sections 3 and 4, proved for (3.1), hold for (4.9) under modified assumptions. The only significant difference is in the proof of Theorem 3.6 for (4.9). Due to condition (4.11) we can show that Lemma 3.2 holds for function $H$. Then by the definition of function $H$ in (4.10), we can represent the $\theta$-EM scheme (4.9) as

$$
H\left(Y_{t_{k+1}}\right)=H\left(Y_{t_{k}}\right)+f\left(Y_{t_{k}}\right) \Delta t+g\left(Y_{t_{k}}\right) \Delta w_{t_{k}} .
$$

Consequently writing $\left\langle H\left(Y_{t_{k+1}}\right), H\left(Y_{t_{k+1}}\right)\right\rangle=\left\|H\left(Y_{t_{k+1}}\right)\right\|^{2}$ we obtain

$$
\begin{aligned}
\left\|H\left(Y_{t_{k+1}}\right)\right\|^{2} & =\left\|H\left(Y_{t_{k}}\right)\right\|^{2}+\left\|f\left(Y_{t_{k}}\right)\right\|^{2} \Delta t^{2}+\left\|g\left(Y_{t_{k}}\right)\right\|^{2} \Delta t \\
& +2\left\langle H\left(Y_{t_{k}}\right), f\left(Y_{t_{k}}\right)\right\rangle \Delta t+\Delta M_{t_{k+1}} \\
& =\left\|H\left(Y_{t_{k}}\right)\right\|^{2}+\left(2\left\langle Y_{t_{k}}, f\left(Y_{t_{k}}\right)\right\rangle+\left\|g\left(Y_{t_{k}}\right)\right\|^{2}\right) \Delta t \\
& +\left[2(1-\theta)\left\langle f_{1}\left(Y_{t_{k}}\right), f_{2}\left(Y_{t_{k}}\right)\right\rangle+\left\|f_{2}\left(Y_{t_{k}}\right)\right\|^{2}+(1-2 \theta)\left\|f_{1}\left(Y_{t_{k}}\right)\right\|^{2}\right] \Delta t^{2}+\Delta M_{t_{k+1}}
\end{aligned}
$$

where

$$
\begin{aligned}
\Delta M_{t_{k+1}} & =\left\|g\left(Y_{t_{k}}\right) \Delta w_{t_{k+1}}\right\|^{2}-\left\|g\left(Y_{t_{k}}\right)\right\|^{2} \Delta t+2\left\langle H\left(Y_{t_{k}}\right), g\left(Y_{t_{k}}\right) \Delta w_{t_{k+1}}\right\rangle \\
& +2\left\langle f\left(Y_{t_{k}}\right) \Delta t, g\left(Y_{t_{k}}\right) \Delta w_{t_{k+1}}\right\rangle .
\end{aligned}
$$

Due to condition (4.12) we have

$$
\left\|H\left(Y_{t_{k+1}}\right)\right\|^{2} \leq\left\|H\left(Y_{t_{k}}\right)\right\|^{2}+2 \alpha \Delta t+2 \beta\left\|Y_{t_{k}}\right\|^{2}+\Delta M_{t_{k+1}} .
$$

The proof can be completed by analogy to the analysis for the $\theta$-EM scheme (3.1). Having boundedness of moments for (4.9) we can show that (4.13) holds in exactly the same way as for $\theta$-EM scheme (3.1).

\subsection{Numerical Example}

In this section we perform a numerical experiment that confirms our theoretical results. Since Multilevel Monte-Carlo simulations provide excellent motivation for our work [12, here we consider the measure of error (4.13) with $s=2$. Although, the case $s=2$ is not covered by our analysis, the numerical 
experiment suggests that Theorem 4.4 still holds. In our numerical experiment, we focus on the error at the endpoint $T=1$, so we let

$$
e_{\Delta t}^{\text {strong }}=\mathbb{E}\left\|x(T)-X_{T}\right\|^{2} .
$$

We consider the SDE (1.4)

$$
d x(t)=\left(\mu-\alpha x(t)^{3}\right) d t+\beta x^{2}(t) d w(t),
$$

where $(\mu, \alpha, \beta)=(0.5,0.2, \sqrt{0.2})$. The assumptions of Theorem 4.4 hold. The $\theta$-EM (3.1) with $\theta=1$, applied to (1.4) writes as

$$
X_{t_{k+1}}=X_{t_{k}}+\left(\mu-\alpha X_{t_{k+1}}^{3}\right) \Delta t+\beta X_{t_{k}}^{2} \Delta w_{t_{k}} .
$$

Since we employ the BEM to approximate (1.4), on each step of the numerical simulation we need to find the inverse of the function $F(x)=\alpha x^{3} \Delta t+x$. In this case we can find the inverse function explicitly and therefore computational complexity is not increased. Indeed, we observe that it is enough to find the real root of the cubic equation

$$
\alpha X_{t_{k+1}}^{3} \Delta t+X_{t_{k+1}}-\left(X_{t_{k}}+\mu \Delta t+\beta X_{t_{k}}^{2} \Delta w_{t_{k}}\right)=0 .
$$

In Figure 1 we plot $e_{\Delta t}^{\text {strong }}$ against $\Delta t$ on a log-log scale. Error bars representing $95 \%$ confidence intervals are denoted by circles. Although we do not know the explicit form of the solution to (1.4), Theorem 4.4

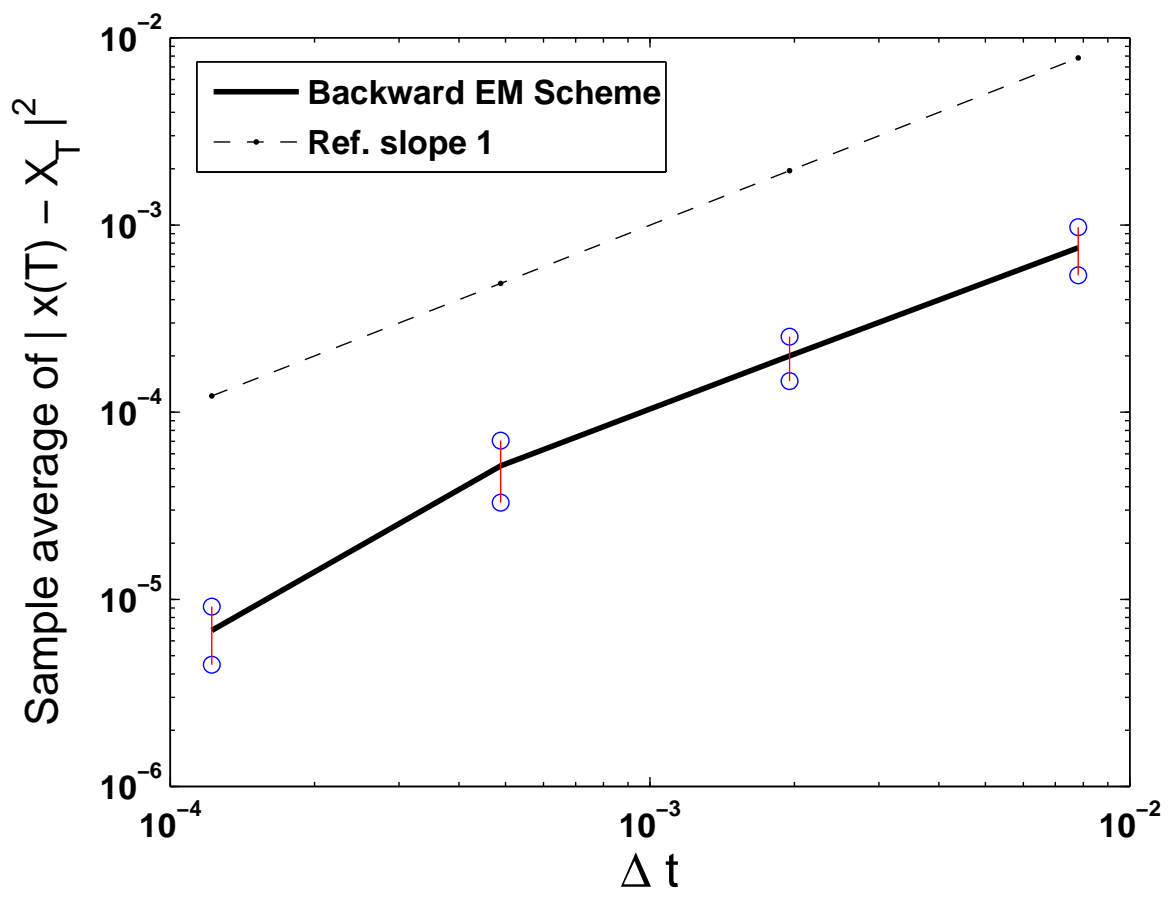

Figure 1: A strong error plot where the dashed line is a reference slope and the continuous line is the extrapolation of the error estimates for the BEM scheme.

guarantees that the BEM (4.15) strongly converges to the true solution. Therefore, it is reasonable to take the BEM with the very small time step $\Delta t=2^{-14}$ as a reference solution. We then compare it to the BEM evaluated with timesteps $\left(2^{1} \Delta t, 2^{3} \Delta t, 2^{5} \Delta t, 2^{7} \Delta t\right)$ in order to estimate the rate of convergence. Since we are using Monte Carlo method, the sampling error decays with a rate of $1 / \sqrt{M}, M$ - is the number of sample paths. We set $M=10000$. From Figure 1 we see that there appears to exist a positive constant such that

$$
e_{\Delta t}^{\text {strong }} \leq C \Delta t \text { for sufficiently small } \Delta t .
$$

Hence, our results are consistent with a strong order of convergence of one-half. 


\section{Stability Analysis}

In this section we examine the globally almost surely asymptotic stability of the $\theta$-EM scheme (3.1). The stability conditions we derive are more related to the mean-square stability, 20, 40. First, we give some preliminary analysis for the SDEs (1.1). We give conditions on the coefficients of the SDEs (1.1) that are sufficient for the globally almost surely asymptotic stability. Later we prove that the $\theta$-EM scheme (3.1) reproduces this asymptotic behavior very well.

\subsection{Continuous Case}

Here we present a simplified version of the stochastic LaSalle Theorem as proved in [42, using the Lyapunov function $V(x)=\|x\|^{2}$.

Theorem 5.1 (Mao et al.). Let Assumption 2.1 hold. We assume that there exists a function $z \in$ $C\left(\mathbb{R}^{n} ; \mathbb{R}_{+}\right)$, such that

$$
\langle x, f(x)\rangle+\frac{1}{2}\|g(x)\|^{2} \leq-z(x)
$$

for all $x \in \mathbb{R}^{n}$. We then have the following assertions:

- For any $x_{0} \in \mathbb{R}^{n}$, the solution $x(t)$ of (1.1) has the properties that

$$
\begin{gathered}
\limsup _{t \rightarrow \infty}\|x(t)\|^{2}<\infty \quad \text { a.s } \quad \text { and } \\
\lim _{t \rightarrow \infty} z(x(t))=0 \quad \text { a.s. }
\end{gathered}
$$

What is more, when $z(x)=0$ if and only if $x=0$ then

$$
\lim _{t \rightarrow \infty} x(t)=0 \quad \text { a.s } \quad \forall x_{0} \in \mathbb{R}^{n} .
$$

\subsection{Almost Sure Stability}

We begin this section with the following Lemma.

Lemma 5.2. Let $Z=\left\{Z_{n}\right\}_{n \in \mathbb{N}}$ be a nonnegative stochastic process with the Doob decomposition $Z_{n}=$ $Z_{0}+A_{n}^{1}-A_{n}^{2}+M_{n}$, where $A^{1}=\left\{A_{n}^{1}\right\}_{n \in \mathbb{N}}$ and $A^{2}=\left\{A_{n}^{2}\right\}_{n \in \mathbb{N}}$ are a.s. nondecreasing, predictable processes with $A_{0}^{1}=A_{0}^{2}=0$, and $M=\left\{M_{n}\right\}_{n \in \mathbb{N}}$ is local $\left\{\mathcal{F}_{n}\right\}_{n \in \mathbb{N}}$-martingale with $M_{0}=0$. Then

$$
\left\{\lim _{n \rightarrow \infty} A_{n}^{1}<\infty\right\} \subseteq\left\{\lim _{n \rightarrow \infty} A_{n}^{2}<\infty\right\} \cap\left\{\lim _{n \rightarrow \infty} Z_{n} \text { exists and is finite }\right\} \quad \text { a.s. }
$$

The original lemma can be found in Liptser and Shiryaev 30. The reader can notice that this lemma combines the Doob decomposition and the martingales convergence theorem. Since we use the Lyapunov function $V(x)=\|x\|^{2}$, our results extend the mean-square stability for linear systems, Higham [15, 16], to a highly non-linear setting. The next theorem demonstrates that there exists a discrete counterpart of Theorem 5.1 for the $\theta$-EM scheme (3.1).

Theorem 5.3. Let Assumptions [2.1, 3.1 and 3.5 hold. Assume that there exists a function $z \in$ 
$C\left(\mathbb{R}^{n} ; \mathbb{R}_{+}\right)$such that for all $x \in \mathbb{R}^{n}$ and for all $\Delta t \in\left(0,(\max \{L, 2 \beta\} \theta)^{-1}\right)$,

$$
\langle x, f(x)\rangle+\frac{1}{2}\|g(x)\|^{2}+\frac{(1-2 \theta)}{2}\|f(x)\|^{2} \Delta t \leq-z(x) .
$$

Then the $\theta$-EM solution defined by (3.1), obeys

$$
\begin{gathered}
\limsup _{k \rightarrow \infty}\left\|X_{t_{k}}\right\|^{2}<\infty \quad \text { a.s. } \\
\lim _{k \rightarrow \infty} z\left(X_{t_{k}}\right)=0 \quad \text { a.s. }
\end{gathered}
$$

If additionally $z(x)=0$ iff $x=0$, then

$$
\lim _{k \rightarrow \infty} X_{t_{k}}=0 \text { a.s. }
$$

Proof. By (3.5) we have

$$
\begin{aligned}
\left\|F\left(X_{t_{k+1}}\right)\right\|^{2}= & \left\|F\left(X_{t_{k}}\right)\right\|^{2}+\left(2\left\langle X_{t_{k}}, f\left(X_{t_{k}}\right)\right\rangle+\left\|g\left(X_{t_{k}}\right)\right\|^{2}\right) \Delta t \\
& +(1-2 \theta)\left\|f\left(X_{t_{k}}\right)\right\|^{2} \Delta t^{2}+\Delta M_{t_{k+1}}
\end{aligned}
$$

where

$$
\begin{aligned}
\Delta M_{t_{k+1}} & =\left\|g\left(X_{t_{k}}\right) \Delta w_{t_{k+1}}\right\|^{2}-\left\|g\left(X_{t_{k}}\right)\right\|^{2} \Delta t+2\left\langle F\left(X_{t_{k}}\right), g\left(X_{t_{k}}\right) \Delta w_{t_{k+1}}\right\rangle \\
& +2\left\langle f\left(X_{t_{k}}\right) \Delta t, g\left(X_{t_{k}}\right) \Delta w_{t_{k+1}}\right\rangle
\end{aligned}
$$

so $\sum_{k=0}^{N} \Delta M_{t_{k+1}}$ is a local martingale due to Assumption 3.5 and Lemma 3.3. Hence, we have obtained the decomposition required to apply Lemma 5.2 that is

$$
\left\|F\left(X_{t_{N+1}}\right)\right\|^{2}=\left\|F\left(X_{t_{0}}\right)\right\|^{2}-\sum_{k=0}^{N} A_{t_{k}} \Delta t+\sum_{k=0}^{N} \Delta M_{k}
$$

where

$$
A_{t_{k}}=-\left(\left(2\left\langle X_{t_{k}}, f\left(X_{t_{k}}\right)\right\rangle+\left\|g\left(X_{t_{k}}\right)\right\|^{2}\right)+(1-2 \theta)\left\|f\left(X_{t_{k}}\right)\right\|^{2} \Delta t\right) .
$$

By condition (5.2), $\sum_{k=0}^{N} A_{t_{k}} \Delta t$ is nondecreasing. Hence by Lemma 5.2 we arrive at

$$
\lim _{k \rightarrow \infty}\left\|F\left(X_{t_{k}}\right)\right\|^{2}<\infty
$$

Consequently, by Lemma 3.2 we arrive at

$$
\limsup _{k \rightarrow \infty}\left\|X\left(t_{k}\right)\right\|^{2}<\infty \quad \text { a.s.. }
$$

By Lemma 5.2,

$$
\sum_{k=0}^{\infty} z\left(X_{t_{k}}\right) \Delta t \leq \sum_{k=0}^{\infty} A_{t_{k}} \Delta t<\infty \quad \text { a.s }
$$

which implies

$$
\lim _{k \rightarrow \infty} z\left(X_{t_{k}}\right)=0 \quad \text { a.s. }
$$




\section{A Proof of Lemma 3.3}

Proof. By (3.6) we obtain

$$
\left\|F\left(X_{t_{k}}\right)\right\|^{2} \leq\left\|F\left(X_{t_{k-1}}\right)\right\|^{2}+2 \alpha \Delta t+2 \beta\left\|X_{t_{k-1}}\right\|^{2} \Delta t+\Delta M_{t_{k}}
$$

where

$$
\begin{aligned}
\Delta M_{t_{k}} & =\left\|g\left(X_{t_{k-1}}\right) \Delta w_{t_{k}}\right\|^{2}-\left\|g\left(X_{t_{k-1}}\right)\right\|^{2} \Delta t+2\left\langle F\left(X_{t_{k-1}}\right), g\left(X_{t_{k-1}}\right) \Delta w_{t_{k}}\right\rangle \\
& +2\left\langle f\left(X_{t_{k-1}}\right) \Delta t, g\left(X_{t_{k-1}}\right) \Delta w_{t_{k}}\right\rangle .
\end{aligned}
$$

Using the basic inequality $\left(a_{1}+a_{2}+a_{3}+a_{4}\right)^{p / 2} \leq 4^{p / 2-1}\left(a_{1}^{p / 2}+a_{2}^{p / 2}+a_{3}^{p / 2}+a_{4}^{p / 2}\right)$, where $a_{i} \geq 0$, we obtain

$$
\left\|F\left(X_{t_{k}}\right)\right\|^{p} \leq 4^{p / 2-1}\left(\left\|F\left(X_{t_{k-1}}\right)\right\|^{p}+(2 \alpha \Delta t)^{p / 2}+(2 \beta)^{p / 2}\left\|X_{t_{k-1}}\right\|^{p} \Delta t^{p / 2}+\left|\Delta M_{t_{k}}\right|^{p / 2}\right) .
$$

As a consequence

$$
\begin{aligned}
\mathbb{E}\left[\left\|F\left(X_{t_{k}}\right)\right\|^{p} \mathbf{1}_{\left[0, \lambda_{m}\right]}(k)\right] \leq & 4^{p / 2-1}\left(\mathbb{E}\left[\left\|F\left(X_{t_{k-1}}\right)\right\|^{p} \mathbf{1}_{\left[0, \lambda_{m}\right]}(k)\right]+(2 \alpha \Delta t)^{p / 2}+\left(2 \beta m^{2} \Delta t\right)^{p / 2}\right. \\
& \left.+\mathbb{E}\left[\left|\Delta M_{t_{k}}\right|^{p / 2} \mathbf{1}_{\left[0, \lambda_{m}\right]}(k)\right]\right) .
\end{aligned}
$$

Due to Assumption 2.1 we can bound $\|F(x)\|^{p}$ and $\|g(x)\|$ for $\|x\|<m$. Whence, there exists a constant $C(m, p)$, such that

$$
\begin{aligned}
& \mathbb{E}\left[\left|\Delta M_{t_{k}}\right|^{p / 2}\right] \mathbf{1}_{\left[0, \lambda_{m}\right]}(k) \\
& \leq 4^{p / 2-1} \mathbb{E}\left[\left\|g\left(X_{t_{k-1}}\right) \Delta w_{k}\right\|^{p}+\left\|g\left(X_{t_{k-1}}\right)\right\|^{p} \Delta t^{p / 2}+\left(2\left\|F\left(X_{t_{k-1}}\right)\right\|\left\|g\left(X_{t_{k-1}}\right) \Delta w_{t_{k}}\right\|\right)^{p / 2}\right. \\
& \left.+\left(2\left\|f\left(X_{t_{k-1}}\right) \Delta t\right\|\left\|g\left(X_{t_{k-1}}\right) \Delta w_{t_{k}}\right\|\right)^{p / 2}\right] \mathbf{1}_{\left[0, \lambda_{m}\right]}(k) \\
& \leq C(m, p) \mathbb{E}\left[1+\left\|g\left(X\left(t_{k-1}\right)\right) \Delta w_{k}\right\|^{p}\right] \mathbf{1}_{\left[0, \lambda_{m}\right]}(k),
\end{aligned}
$$

By Hölder's inequality

$$
\begin{aligned}
& \mathbb{E}\left[\left|\Delta M_{t_{k}}\right|^{p / 2}\right] \mathbf{1}_{\left[0, \lambda_{m}\right]}(k) \\
& \leq C(m, p)\left[1+\left(\mathbb{E}\left[\left\|g\left(X_{t_{k-1}}\right)\right\|^{2 p} \mathbf{1}_{\left[0, \lambda_{m}\right]}(k)\right]\right)^{1 / 2}\left(\mathbb{E}\left\|\Delta w_{t_{k-1}}\right\|^{2 p}\right)^{1 / 2}\right] .
\end{aligned}
$$

Hence

$$
\mathbb{E}\left[\left\|F\left(X_{t_{k}}\right)\right\|^{p} \mathbf{1}_{\left[0, \lambda_{m}\right]}(k)\right] \leq C(m, p)\left(1+\left(\mathbb{E}\left[\left\|g\left(X_{t_{k-1}}\right)\right\|^{p} \mathbf{1}_{\left[0, \lambda_{m}\right]}(k)\right]^{2}\right)^{1 / 2}\left(\mathbb{E}\left\|\Delta w_{t_{k-1}}\right\|^{2 p}\right)^{1 / 2}\right)
$$


Since there exists a positive constant $C(p)$, such that $\mathbb{E}\left\|\Delta w_{t_{k-1}}\right\|^{2 p}<C(p)$, we obtain

$$
\mathbb{E}\left[\left\|F\left(X_{t_{k}}\right)\right\|^{p} \mathbf{1}_{\left[0, \lambda_{m}\right]}(k)\right]<C(m, p) .
$$

We conclude the assertion by applying Lemma 3.2 .

\section{B Proof of Theorem 4.2}

Proof. By the Itô formula

$$
\begin{aligned}
\left\|\hat{X}\left(T \wedge \vartheta_{m}\right)\right\|^{2} & =\left\|x_{0}\right\|^{2}+\int_{0}^{T \wedge \vartheta_{m}}\left(2\left\langle\hat{X}(s), f\left(X_{\eta(s)}\right)\right\rangle+\operatorname{trace}\left[g^{T}\left(X_{\eta(s)}\right) I_{n \times n} g\left(X_{\eta(s)}\right)\right]\right) d s \\
& +2 \int_{0}^{T \wedge \vartheta_{m}}\left\langle\hat{X}(s), g\left(X_{\eta(s)}\right)\right\rangle d w(s) \\
& =\left\|x_{0}\right\|^{2}+\int_{0}^{T \wedge \vartheta_{m}}\left(2\left\langle\hat{X}(s)-X_{\eta(s)}+X_{\eta(s)}, f\left(X_{\eta(s)}\right)\right\rangle+\left\|g\left(X_{\eta(s)}\right)\right\|^{2}\right) d s \\
& +2 \int_{0}^{T \wedge \vartheta_{m}}\left\langle\hat{X}(s), g\left(X_{\eta(s)}\right)\right\rangle d w(s) \\
& \leq\left\|x_{0}\right\|^{2}+\int_{0}^{T \wedge \vartheta_{m}}\left(2\left\langle X_{\eta(s)}, f\left(X_{\eta(s)}\right)\right\rangle+\left\|g\left(X_{\eta(s)}\right)\right\|^{2}\right) d s \\
& +2 \int_{0}^{T \wedge \vartheta_{m}}\left\|\hat{X}(s)-X_{\eta(s)}\right\|\left\|f\left(X_{\eta(s)}\right)\right\| d s \\
& +2 \int_{0}^{T \wedge \vartheta_{m}}\left\langle\hat{X}(s), g\left(X_{\eta(s)}\right)\right\rangle d w(s) .
\end{aligned}
$$

By Assumption 2.1, for $\|x\| \leq m$

$$
\begin{gathered}
\|f(x)\|^{2} \leq 2\left(\|f(x)-f(0)\|^{2}+\|f(0)\|^{2}\right) \leq 2\left(C(m)\|x\|^{2}+\|f(0)\|^{2}\right), \\
\|g(x)\|^{2} \leq 2\left(\|g(x)-g(0)\|^{2}+\|g(0)\|^{2}\right) \leq 2\left(C(m)\|x\|^{2}+\|g(0)\|^{2}\right),
\end{gathered}
$$

and

$\mathbb{E}\left\|\hat{X}\left(T \wedge \vartheta_{m}\right)\right\|^{2} \leq\left\|x_{0}\right\|^{2}+2 \alpha T+2 \beta \mathbb{E} \int_{0}^{T \wedge \vartheta_{m}}\left\|X_{\eta(s)}-\hat{X}(s)+\hat{X}(s)\right\|^{2} d s+C(m) \mathbb{E} \int_{0}^{T \wedge \vartheta_{m}}\left\|X_{\eta(s)}-\hat{X}(s)\right\| d s$.

Using the basic inequality $(a-b+c)^{2} \leq 2\left(\|a-b\|^{2}+\|c\|^{2}\right)$ and the fact that $\int_{0}^{T \wedge \vartheta_{m}}\left\|X_{\eta(s)}-\hat{X}(s)\right\|^{2} d s \leq$ $C(m) \int_{0}^{T \wedge \vartheta_{m}}\left\|X_{\eta(s)}-\hat{X}(s)\right\| d s$, we obtain

$$
\begin{aligned}
\mathbb{E}\left\|\hat{X}\left(T \wedge \vartheta_{m}\right)\right\|^{2} \leq & \left\|x_{0}\right\|^{2}+2 \alpha T+4 \beta \int_{0}^{T} \mathbb{E}\left\|\hat{X}\left(s \wedge \vartheta_{m}\right)\right\|^{2} d s \\
& +4 \beta \mathbb{E} \int_{0}^{T \wedge \vartheta_{m}}\left\|X_{\eta(s)}-\hat{X}(s)\right\|^{2} d s+C(m) \mathbb{E} \int_{0}^{T \wedge \vartheta_{m}}\left\|X_{\eta(s)}-\hat{X}(s)\right\| d s \\
\leq & \left\|x_{0}\right\|^{2}+2 \alpha T+4 \beta \int_{0}^{T} \mathbb{E}\left\|\hat{X}\left(s \wedge \vartheta_{m}\right)\right\|^{2} d s+C(m)(4 \beta+1) \mathbb{E} \int_{0}^{T \wedge \vartheta_{m}}\left\|X_{\eta(s)}-\hat{X}(s)\right\| d s .
\end{aligned}
$$


Since $\lambda_{m} \geq \vartheta_{m}$ a.s., Lemma 4.1 gives the following bound

$$
\mathbb{E} \int_{0}^{T \wedge \vartheta_{m}}\left\|X_{\eta(s)}-\hat{X}_{\eta(s)}\right\| d s \leq C(m, T) \Delta t .
$$

To bound the term $\mathbb{E} \int_{0}^{T \wedge \vartheta_{m}}\left\|\hat{X}_{\eta(s)}-\hat{X}(s)\right\| d s$ in (B.3), first we observe that

$$
\left\|\hat{X}_{\eta(s)}-\hat{X}(s)\right\| \mathbf{1}_{\left[t_{k}, t_{k+1}\right)}(s)=\left\|\int_{t_{k}}^{s} f\left(X_{t_{k}}\right) d h+\int_{t_{k}}^{s} g\left(X_{t_{k}}\right) d w(h)\right\| \mathbf{1}_{\left[t_{k}, t_{k+1}\right)}(s) .
$$

Then, by (B.1) and (B.2)

$$
\mathbb{E} \int_{0}^{T \wedge \vartheta_{m}}\left\|\hat{X}_{\eta(s)}-\hat{X}(s)\right\| d s \leq C(m, T) \Delta t^{\frac{1}{2}}
$$

where $C(m, T)>0$ is constant. Combining (B.4) and (B.5) leads us to

$$
\begin{aligned}
E \int_{0}^{T \wedge \vartheta_{m}}\left\|X_{\eta(s)}-\hat{X}(s)\right\| d s & \leq \mathbb{E} \int_{0}^{T \wedge \vartheta_{m}}\left\|\hat{X}_{\eta(s)}-\hat{X}(s)\right\| d s \\
& +\mathbb{E} \int_{0}^{T \wedge \vartheta_{m}}\left\|X_{\eta(s)}-\hat{X}_{\eta(s)}\right\| d s \\
& \leq C(m, T) \Delta t^{\frac{1}{2}} .
\end{aligned}
$$

Therefore

$$
\mathbb{E}\left\|\hat{X}\left(T \wedge \vartheta_{m}\right)\right\|^{2} \leq\left\|x_{0}\right\|^{2}+2 \alpha T+C(m, T) \Delta t^{\frac{1}{2}}+4 \beta \int_{0}^{T} \mathbb{E}\left\|\hat{X}\left(s \wedge \vartheta_{m}\right)\right\|^{2} d s .
$$

By Gronwall's inequality

$$
\mathbb{E}\left\|\hat{X}\left(T \wedge \vartheta_{m}\right)\right\|^{2} \leq\left[\left\|x_{0}\right\|^{2}+2 \alpha T+C(m, T) \Delta t^{\frac{1}{2}}\right] \exp (4 \beta T) .
$$

Now we will find the lower bound for $\left\|\hat{X}\left(\vartheta_{m}\right)\right\|^{2}$. From the definition of the stopping time $\vartheta_{m}$, if $\inf \{t>0:\|\hat{X}(t)\| \geq m\} \leq \inf \left\{t>0:\left\|X_{\eta(t)}\right\|>m\right\}$ then $\left\|\hat{X}\left(\vartheta_{m}\right)\right\|^{2}=m^{2}$. In the alternative case, where $\inf \{t>0:\|\hat{X}(t)\| \geq m\}>\inf \left\{t>0:\left\|X_{\eta(t)}\right\|>m\right\}$, we have $\left\|X_{\vartheta_{m}}\right\|^{2}>m^{2}$. From Lemmas 3.2 and 4.1 we arrive at

$$
\left\|\hat{X}\left(\vartheta_{m}\right)\right\|^{2} \geq \frac{1}{2}\left((1-2 \beta \theta \Delta t)\left\|X_{\vartheta_{m}}\right\|^{2}-2 \theta \alpha \Delta t\right)-\left\|\theta f\left(x_{0}\right) \Delta t\right\|^{2} .
$$

Hence there exist positive constants $c_{1}$ and $c_{2}$ such that

$$
\left\|\hat{X}\left(\vartheta_{m}\right)\right\|^{2}>c_{1} m^{2}-c_{2} \Delta t
$$

We have

$$
\mathbb{E}\left\|\hat{X}\left(T \wedge \vartheta_{m}\right)\right\|^{2} \geq \mathbb{E}\left[\mathbf{1}_{\left\{\vartheta_{m}<T\right\}}\left\|\hat{X}\left(\vartheta_{m}\right)\right\|^{2}\right] \geq \mathbb{P}\left(\vartheta_{m}<T\right)\left(c_{1} m^{2}-c_{2} \Delta t\right) .
$$

which implies that

$$
\mathbb{P}\left(\vartheta_{m}<T\right) \leq \frac{\left[\left\|x_{0}\right\|^{2}+2 \alpha T+C(m, T) \Delta t^{1 / 2}\right] \exp (4 \beta T)}{c_{1} m^{2}-c_{2} \Delta t} .
$$

Now, for any given $\epsilon>0$, we choose $N_{0}$ such that for any $m \geq N_{0}$

$$
\frac{\left[\left\|x_{0}\right\|^{2}+2 \alpha T\right] \exp (4 \beta T)}{c_{1} m^{2}-c_{2} \Delta t} \leq \frac{\epsilon}{2} .
$$


Then, we can choose $\Delta t_{0}=\Delta t_{0}(m)$, such that for any $\Delta t \leq \Delta t_{0}$

$$
\frac{\exp (4 \beta T) C(m, T) \Delta t^{1 / 2}}{c_{1} m^{2}-c_{2} \Delta t} \leq \frac{\epsilon}{2},
$$

whence $\mathbb{P}\left(\vartheta_{m}<T\right) \leq \epsilon$ as required.

\section{Proof of Lemma 4.3}

Proof. It is useful to observe that since the constant $C(T, m)$ in (4.5) depends on $m$, we can prove the theorem in a similar way as in the classical case where coefficients $f$ and $g$ in (1.1) obey the global Lipschitz condition [18, 26]. Nevertheless, for completeness of the exposition we present the sketch of the proof. For any $T_{1} \in[0, T]$, by Hölder's and Burkholder-Davis-Gundy's inequalities

$$
\begin{aligned}
& \mathbb{E}\left[\sup _{0 \leq t \leq T_{1}}\left\|\hat{X}\left(t \wedge \theta_{m}\right)-x\left(t \wedge \theta_{m}\right)\right\|^{2}\right] \\
& \leq 2\left(T \mathbb{E} \int_{0}^{T_{1} \wedge \theta_{m}}\left\|f\left(X_{\eta(s)}\right)-f(x(s))\right\|^{2} d s+4 \mathbb{E} \int_{0}^{T_{1} \wedge \theta_{m}}\left\|g\left(X_{\eta(s)}\right)-g(x(s))\right\|^{2} d s\right)
\end{aligned}
$$

By Assumption 2.1 there exists a constant $C(m)$

$$
\begin{aligned}
& \mathbb{E}\left[\sup _{0 \leq t \leq T_{1}}\left\|\hat{X}\left(t \wedge \theta_{m}\right)-x\left(t \wedge \theta_{m}\right)\right\|^{2}\right] \\
& \leq 2 C(m)\left(T \mathbb{E} \int_{0}^{T_{1} \wedge \theta_{m}}\left\|X_{\eta(s)}-x(s)\right\|^{2} d s+4 \mathbb{E} \int_{0}^{T_{1} \wedge \theta_{m}}\left\|X_{\eta(s)}-x(s)\right\|^{2} d s\right) \\
& \leq 4 C(m)\left(T \mathbb{E} \int_{0}^{T_{1} \wedge \theta_{m}}\left[\|\hat{X}(s)-x(s)\|^{2}+\left\|X_{\eta(s)}-\hat{X}(s)\right\|^{2}\right] d s\right. \\
& \left.+4 \mathbb{E} \int_{0}^{T_{1} \wedge \theta_{m}}\left[\|\hat{X}(s)-x(s)\|^{2}+\left\|X_{\eta}(s)-\hat{X}(s)\right\|^{2}\right] d s\right) \\
& \leq 4 C(m)(T+4) \mathbb{E} \int_{0}^{T_{1}}\left\|\hat{X}\left(s \wedge \theta_{m}\right)-x\left(s \wedge \theta_{m}\right)\right\|^{2} d s \\
& +4 C(m)(T+4) \mathbb{E} \int_{0}^{T_{1} \wedge \theta_{m}}\left\|X_{\eta}(s)-\hat{X}(s)\right\|^{2} d s .
\end{aligned}
$$

By the same reasoning which gave estimate (B.6), we can deduce that

$$
\mathbb{E} \int_{0}^{T_{1} \wedge \theta_{m}}\left\|X_{\eta}(s)-\hat{X}(s)\right\|^{2} d s \leq C(m, T) \Delta t .
$$

Hence

$$
\begin{aligned}
& \mathbb{E}\left[\sup _{0 \leq t \leq T_{1}}\left\|\hat{X}\left(t \wedge \theta_{m}\right)-x\left(t \wedge \theta_{m}\right)\right\|^{2}\right] \\
& \leq 4 C(m)(T+4) C(m, T) \Delta t+4 C(m)(T+4) \mathbb{E}\left[\int_{0}^{T_{1}} \sup _{0 \leq t \leq s}\left\|\hat{X}\left(t \wedge \theta_{m}\right)-x\left(t \wedge \theta_{m}\right)\right\|^{2} d s\right] .
\end{aligned}
$$

The statement of the theorem holds by the Gronwall inequality. 


\section{References}

[1] D.H. Ahn and B. Gao. A parametric nonlinear model of term structure dynamics. Review of Financial Studies, 12(4):721, 1999.

[2] Y. Ait-Sahalia. Testing continuous-time models of the spot interest rate. Review of Financial Studies, 9(2):385-426, 1996.

[3] A. Bahar and X. Mao. Stochastic delay population dynamics. International Journal of Pure and Applied Mathematics, 11:377-400, 2004.

[4] C.T.H. Baker and E. Buckwar. Exponential stability in p-th mean of solutions, and of convergent Euler-type solutions, of stochastic delay differential equations. Journal of Computational and Applied Mathematics, 184(2):404-427, 2005.

[5] A. Berkaoui, M. Bossy, and A. Diop. Euler scheme for SDEs with non-Lipschitz diffusion coefficient: strong convergence. ESAIM: Probability and Statistics, 12:1-11, 2007.

[6] M. Broadie, P. Glasserman, and S. Kou. A continuity correction for discrete barrier options. Mathematical Finance, 7(4):325-349, 1997.

[7] F.M. Buchmann. Simulation of stopped diffusions. Journal of Computational Physics, 202(2):446462, 2005.

[8] J.Y. Campbell, A.W. Lo, A.C. MacKinlay, and R.F. Whitelaw. The econometrics of financial markets. Macroeconomic Dynamics, 2(04):559-562, 1998.

[9] K.C Chan, G.A. Karolyi, F.A. Longstaff, and A.B. Sanders. An empirical comparison of alternative models of the short-term interest rate. The journal of finance, 47(3):1209-1227, 1992.

[10] A. Friedman. Stochastic differential equations and applications. Academic Press, 1976.

[11] T.C. Gard. Introduction to Stochastic Differential Equations. Marcel Dekker, New York, 1988.

[12] M.B. Giles. Multilevel monte carlo path simulation. Operations Research-Baltimore, 56(3):607-617, 2008.

[13] J.K. Hale and S.M.V. Lunel. Introduction to Functional Differential Equations. Springer Verlag, 1993.

[14] S.L. Heston. A simple new formula for options with stochastic volatility. Course notes of Washington University in St. Louis, Missouri, 1997.

[15] D.J. Higham. A-stability and stochastic mean-square stability. BIT Numerical Mathematics, 40(2):404-409, 2000.

[16] D.J. Higham. Mean-square and asymptotic stability of the stochastic theta method. SIAM Journal on Numerical Analysis, pages 753-769, 2001.

[17] D.J. Higham and X. Mao. Convergence of Monte Carlo simulations involving the mean-reverting square root process. Journal of Computational Finance, 8(3):35, 2005.

[18] D.J. Higham, X. Mao, and A.M. Stuart. Strong convergence of Euler-type methods for nonlinear stochastic differential equations. SIAM Journal on Numerical Analysis, 40(3):1041-1063, 2002.

[19] D.J. Higham, X. Mao, and A.M. Stuart. Exponential mean-square stability of numerical solutions to stochastic differential equations. LMS J. Comput. Math, 6:297-313, 2003.

[20] D.J. Higham, X. Mao, and C. Yuan. Almost sure and moment exponential stability in the numerical simulation of stochastic differential equations. SIAM Journal on Numerical Analysis, 45(2):592-609, 2008 .

[21] Y. Hu. Semi-implicit Euler-Maruyama scheme for stiff stochastic equations. Progress in Probability, pages $183-202,1996$. 
[22] M. Hutzenthaler, A. Jentzen, and P.E. Kloeden. Strong convergence of an explicit numerical method for sdes with non-globally lipschitz continuous coefficients. to appear in The Annals of Applied Probability, 2010.

[23] M. Hutzenthaler, A. Jentzen, and P.E. Kloeden. Strong and weak divergence in finite time of Euler's method for stochastic differential equations with non-globally Lipschitz continuous coefficients. Proceedings of the Royal Society A: Mathematical, Physical and Engineering Science, 467(2130):1563, 2011.

[24] R.Z. Khasminski. Stochastic Stability of Differential Equations. Kluwer Academic Pub, 1980.

[25] P.E. Kloeden and A. Neuenkirch. The pathwise convergence of approximation schemes for stochastic differential equations. Journal of Computation and Mathematics, 10:235-253, 2007.

[26] P.E. Kloeden and E. Platen. Numerical Solution of Stochastic Differential Equations. Springer, 1992.

[27] J.P. LaSalle. Stability theory for ordinary differential equations. J. Differential Equations, 4(1):5765, 1968.

[28] J.P. LaSalle and Z. Artstein. The Stability of Dynamical Systems. Society for Industrial Mathematics, 1976.

[29] A.L. Lewis. Option Valuation Under Stochastic Volatility. Finance Press, 2000.

[30] R.S. Liptser and A.N. Shiryayev. Theory of Martingales. Kluwer Academic Publishers, 1989.

[31] J.J. Lucia and E.S. Schwartz. Electricity prices and power derivatives: Evidence from the nordic power exchange. Review of Derivatives Research, 5(1):5-50, 2002.

[32] R. Mannella. Absorbing boundaries and optimal stopping in a stochastic differential equation. Physics Letters A, 254(5):257-262, 1999.

[33] X. Mao. Stochastic versions of the LaSalle theorem. Journal of Differential Equations, 153(1):175195, 1999.

[34] X. Mao. Stochastic Differential Equations and Applications. Horwood Pub Ltd, 2007.

[35] X. Mao, G. Marion, and E. Renshaw. Environmental Brownian noise suppresses explosions in population dynamics. Stochastic Process. Appl, 97(1):95-110, 2002.

[36] X. Mao, S. Sabanis, and E. Renshaw. Asymptotic behaviour of the stochastic Lotka-Volterra model. Journal of Mathematical Analysis and Applications, 287(1):141-156, 2003.

[37] X. Mao and L. Szpruch. Strong convergence rates for backward EulerMaruyama method for nonlinear dissipative-type stochastic differential equations with super-linear diffusion coefficients. to appear in Stochastics, 2012.

[38] X. Mao and C. Yuan. Stochastic Differential Equations with Markovian Switching. Imperial College Press, 2006.

[39] X. Mao, C. Yuan, and G. Yin. Approximations of Euler-Maruyama type for stochastic differential equations with Markovian switching, under non-Lipschitz conditions. Journal of Computational and Applied Mathematics, 205(2):936-948, 2007.

[40] J.C. Mattingly, A.M. Stuart, and DJ Higham. Ergodicity for sdes and approximations: locally lipschitz vector fields and degenerate noise. Stochastic processes and their applications, 101(2):185232, 2002.

[41] S. Pang, F. Deng, and X. Mao. Asymptotic properties of stochastic population dynamics. Dynamics of Continuous, Discrete and Impulsive Systems Series A, 15:603-620, 2008.

[42] Y. Shen, Q. Luo, and X. Mao. The improved LaSalle-type theorems for stochastic functional differential equations. Journal of Mathematical Analysis and Applications, 318(1):134-154, 2006.

[43] L. Szpruch, X. Mao, D.J. Higham, and J. Pan. Numerical simulation of a strongly nonlinear AitSahalia-type interest rate model. BIT Numerical Mathematics, pages 1-21. 